\title{
Structural and mutational analysis of the interaction between the Middle-East respiratory syndrome coronavirus (MERS-CoV) papain-like protease and human ubiquitin
}

\author{
Jian Lei ${ }^{1,2}$, Rolf Hilgenfeld ${ }^{1,2}$
}

1. Institute of Biochemistry, Center for Structural and Cell Biology in Medicine, University of Lübeck, 23562

Lübeck, Germany

2. German Center for Infection Research (DZIF), University of Lübeck, 23562 Lübeck, Germany

The papain-like protease $\left(\mathrm{PL}^{\text {pro }}\right.$ ) of Middle-East respiratory syndrome coronavirus (MERS-CoV) has proteolytic, deubiquitinating, and deISGylating activities. The latter two are involved in the suppression of the antiviral innate immune response of the host cell. To contribute to an understanding of this process, we present here the X-ray crystal structure of a complex between MERS-CoV $\mathrm{PL}^{\text {pro }}$ and human ubiquitin (Ub) that is devoid of any covalent linkage between the two proteins. Five regions of the $\mathrm{PL}^{\text {pro }}$ bind to two areas of the $\mathrm{Ub}$. The $\mathrm{C}$-terminal five residues of $\mathrm{Ub}$, RLRGG, are similar to the $\mathrm{P} 5-\mathrm{P} 1$ residues of the polyprotein substrates of the $\mathrm{PL}^{\text {pro }}$ and are responsible for the major part of the interaction between the two macromolecules. Through sitedirected mutagenesis, we demonstrate that conserved Asp165 and non-conserved Asp164 are important for the catalytic activities of MERS-CoV PL ${ }^{\text {pro }}$. The enzyme appears not to be optimized for catalytic efficiency; thus, replacement of Phe269 by Tyr leads to increased peptidolytic and deubiquitinating activities. Ubiquitin binding by MERS-CoV PL ${ }^{\text {pro }}$ involves remarkable differences compared to the corresponding complex with SARS-CoV $\mathrm{PL}^{\text {pro }}$. The structure and the mutational study help understand common and unique features of the deubiquitinating activity of MERS-CoV $\mathrm{PL}^{\text {pro }}$.

KEYWORDS coronavirus; Middle-East respiratory syndrome (MERS); papain-like protease; ubiquitin; deubiquitinase

\section{INTRODUCTION}

To date, six coronaviruses infecting humans have been characterized. Infections with human coronaviruses (HCoVs) 229E (Hamre \& Procknow, 1966), OC43 (McIntosh et al., 1967), NL63 (van der Hoek et al., 2004), and HKU1 (Woo et al., 2005) cause relatively mild symptoms in most cases, whereas severe acute res-

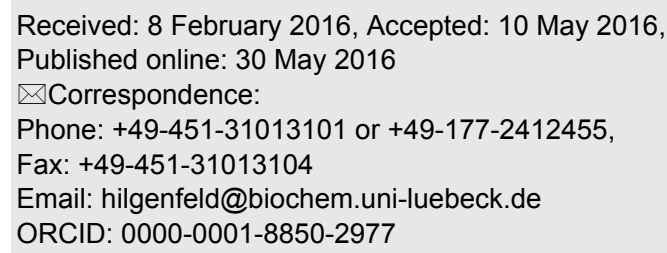

piratory syndrome coronavirus (SARS-CoV; Drosten et al., 2003; Ksiazek et al., 2003; Kuiken et al., 2003; Peiris et al., 2003) and Middle-East respiratory syndrome coronavirus (MERS-CoV; Zaki et al., 2012) are connected with severe respiratory-tract infection and, in particular in case of MERS-CoV, acute renal failure (Eckerle et al., 2013 ), leading to high case-fatality rates of $\sim 10$ and $\sim 35 \%$, respectively. In spite of 13 years of research on SARS-CoV (Hilgenfeld \& Peiris, 2013), no approved drugs or vaccines are available for the treatment or prevention of coronavirus infection (Wang et al., 2016). This is mainly due to the fact that although these emerging viruses have devastating effects on those infected, the absolute numbers of cases ( $~ 8000$ for SARS, 1733 so far for MERS; (http://www.who.int)) imply that the de- 
velopment of specific antivirals is very likely not commercially viable. On the other hand, the global risk posed by MERS-CoV must not be underestimated. Since its discovery in September 2012, the number of MERS cases reported has been rising steadily, with some intermittent peaks connected to hospital outbreaks in Saudi Arabia (Assiri et al., 2013). Man-to-man transmission of MERS-CoV has also been impressively demonstrated by the recent outbreak of MERS in South Korea, which was traced back to a single traveller from the Arab peninsula (Butler, 2015). Therefore, it is imperative that academic laboratories help increase the preparedness against a possible MERS-CoV pandemic by characterizing antiviral drug targets and by identifying lead compounds interfering with them.

In order to successfully infect humans, a virus has to meet at least two conditions: 1), it should maintain a sufficiently correct replication of its genetic material; 2), it should inhibit the host antiviral response. The papainlike protease $\left(\mathrm{PL}^{\mathrm{pro}}\right)$ of MERS-CoV (or SARS-CoV) is involved in both of these tasks (Yang et al., 2013; Barretto et al, 2005). The $\mathrm{PL}^{\mathrm{pro}}$ is a domain located in the middle part of the largest non-structural protein, Nsp3, of MERS-CoV (or SARS-CoV). It is responsible for releasing Nsp1, Nsp2, and Nsp3 from the polyproteins 1a (ppla) and $1 \mathrm{ab}$ (pplab), an essential step of replication (Harcourt et al., 2004). Like its SARS-CoV counterpart, the MERS-CoV PL ${ }^{\text {pro }}$ also has deubiquitinating (DUB) and deISGylating activities in vivo as well as in vitro (Yang et al., 2013; Mielech et al., 2014; Lei et al., 2014; Baez-Santos et al., 2014b). K48- and K63-polyubiquitin poly (Ub) and ISG15 (interferon-stimulated gene 15) conjugated targets are usually involved in host innate immune regulation (Liu et al., 2005; Maringer \& Fernandez-Sesma, 2014). The $\mathrm{PL}^{\text {pro }}$ has the ability to digest K48- and K63-linked polyUb chains and to remove ISG15 from ISG15-linked proteins (Baez-Santos et al., 2014 b), thereby interrupting the signalling pathways leading to the innate immune response. Thus, the $\mathrm{PL}^{\text {pro }}$ can block the activation of IFN regulatory factor 3 (IRF3) (Yang et al., 2013) and subsequently the production of interferon $\beta$ (IFN $\beta$ ) (Mielech et al., 2014). Interestingly, MERS-CoV PL ${ }^{\text {pro }}$ shows a similar cleavage rate for K48- and K63-linked polyUb chains, while the SARS-CoV enzyme prefers K48- over K63-linked chains (Baez-Santos et al., 2014b). The former enzyme degrades a polyUb chain by removing mono-Ubs, whereas the latter cleaves di-Ub units off the polyUb chain (Bekes et al., 2015).

We have reported the first crystal structure of the MERS-CoV PL ${ }^{\text {pro }}$ (Lei et al., 2014). Later, two other groups also described the structure of this enzyme (Bailey-Elkin et al., 2014; Lee et al., 2015). The structure of $\mathrm{PL}^{\text {pro }}$ can be divided into two parts: a ubiquitin- like (Ubl) domain and a catalytic domain with thumb, palm, and fingers subdomains. The overall fold of MERS-CoV PL ${ }^{\text {pro }}$ is not only similar to that of SARSCoV PL ${ }^{\text {pro }}$, but also to that of several human ubiquitinspecific proteases (USPs) (Hu et al., 2005). In 2014, the $\mathrm{X}$-ray structure of the complex of SARS-CoV PL ${ }^{\text {pro }}$ with ubiquitin has been reported (Chou et al., 2014; Ratia et al., 2014). Several key residues (such as Glu168 or Tyr265) of SARS-CoV PL ${ }^{\text {pro }}$ that are important for ubiquitin recognition (Chou et al., 2014; Ratia et al., 2014), are not conserved in MERS-CoV PL ${ }^{\text {pro }}$. Bailey-Elkin et al. (2014) described the structure of an artificially linked, covalent complex between ubiquitin and MERS-CoV $\mathrm{PL}^{\text {pro }}$. Here, we present the crystal structure of a noncovalent complex between the two proteins and a mutational study of the interactions involved. For these studies, we used the Cys111Ser active-site variant of MERSCoV PL ${ }^{\text {pro }}$.

\section{MATERIALS AND METHODS}

\section{Recombinant production of MERS-CoV PL ${ }^{\text {pro }}$ and its variants}

The PL ${ }^{\text {pro }}$ of MERS-CoV (strain $2 c E M C / 2012$; GenBank no. AFV09327.1) contains 320 residues, from Gln1482 to Asp1801 of ppla/1ab. For simplification, Gln1482 was renumbered into Gln1 here. The DNA plasmid coding for MERS-CoV PL ${ }^{\text {pro }}$ was produced earlier (Lei et al., 2014).

The MERS-CoV PL ${ }^{\text {pro }}$ C111S, D164E, D164A, D165E, D165A, and F269Y variants were produced using the same strategy that we described before (Lei et al., 2014). All primers for these variants are listed in Supplemental Table S1. All DNA plasmids coding for the altered $\mathrm{PL}^{\text {pro }}$ were verified by sequencing.

Genes coding for wild-type (WT) MERS-CoV PL ${ }^{\text {pro }}$ and for its variants were expressed and the corresponding proteins were purified according to our previous description (Lei et al., 2014).

\section{Recombinant production of SARS-CoV PL ${ }^{\text {pro }}$}

The PL ${ }^{\text {pro }}$ of SARS-CoV (Strain TOR2; GenBank no. AY274119.3) comprises 319 amino-acid residues, corresponding to Glu1541 to Tyr1859 of pp1a/1ab. A gene coding for the SARS-CoV PL ${ }^{\text {pro }}$ was amplified by PCR with the following two primers 5'-CTAGCTAGCGAGGTTA AGACTATAAAAGTGTTC-3' (forward) and 5'CCGCTCGAGTTAATACGACACAGGCTTGATGGTTG TAG-3' (reverse). The PCR product was digested by Nhe I and Xho I, then was ligated into the pET-28a plasmid (Novagen). The recombinant plasmid DNA was verified by sequencing. Expression of the gene construct coding for SARS-CoV PL ${ }^{\text {pro }}$ and purification of the protein were 
performed according to the procedure described for MERS-CoV PL ${ }^{\text {pro }}$ (Lei et al., 2014).

Crystallization of MERS-CoV PL ${ }^{\text {pro }}$ with ubiquitin Purified MERS-CoV PL ${ }^{\text {pro }}$ (WT) and a variant that had the active-site Cys111 replaced by Ser $\left(\mathrm{PL}^{\mathrm{pro}}(\mathrm{C} 111 \mathrm{~S})\right)$ were both concentrated to $24 \mathrm{mg} / \mathrm{mL}$ in $20 \mathrm{mmol} / \mathrm{L}$ Tris-HCl, $150 \mathrm{mmol} / \mathrm{L} \mathrm{NaCl}, \mathrm{pH} 8.8,10 \mathrm{mmol} / \mathrm{L} \beta$-mercaptoethanol (BME). Human ubiquitin (BostonBiochem) was dissolved to $6 \mathrm{mg} / \mathrm{mL}$ in $20 \mathrm{mmol} / \mathrm{L}$ Tris- $\mathrm{HCl}, 150$ $\mathrm{mmol} / \mathrm{L} \mathrm{NaCl}, \mathrm{pH}$ 8.8. $500 \mu \mathrm{L} \mathrm{PL}^{\text {pro }}$ (WT) or PL ${ }^{\text {pro }}$ (C111S) were mixed with $500 \mu \mathrm{L}$ ubiquitin $(\sim 1: 1$ molar ratio) at 4 ${ }^{\circ} \mathrm{C}$ overnight. The complex of $\mathrm{PL}^{\text {pro }}$ (WT)-Ub or PL ${ }^{\text {pro }}$ (C111S)-Ub was purified by gel filtration $\left(\mathrm{HiLoad}^{\mathrm{TM}}\right.$ $16 / 60 \mathrm{~S} 200$ column, GE Healthcare) in $20 \mathrm{mmol} / \mathrm{L}$ Tris$\mathrm{HCl}, 150 \mathrm{mmol} / \mathrm{L} \mathrm{NaCl}, \mathrm{pH} 8.8$ the next day. The final concentration of $\mathrm{PL}^{\text {pro }}$ (WT)-Ub or $\mathrm{PL}^{\text {pro }}$ (C111S)-Ub was $\sim 12 \mathrm{mg} / \mathrm{mL}$. The two complexes were crystallized using the sitting-drop method and a Phoenix crystallization robot (Art Robbins) at $18{ }^{\circ} \mathrm{C} .0 .25 \mu \mathrm{L}$ of protein and $0.25 \mu \mathrm{L}$ of reservoir were mixed and equilibrated against $75 \mu \mathrm{L}$ reservoir. Screening kits Index ${ }^{\mathrm{TM}}$, SaltRx ${ }^{\mathrm{TM}}$, PEG $\mathrm{Rx}^{\mathrm{TM}} 1 \& 2$, PEG/Ion ${ }^{\mathrm{TM}} 1 \& 2$ (Hampton Research), and Structure Screen 1 MD1-01, Structure Screen 2 MD1-02 (Molecular Dimensions) were used. Crystals of PL ${ }^{\text {pro }}$ (C111S)-Ub were observed under condition No. 9 of MD1-01, whereas no crystal of PL ${ }^{\text {pro }}$ (WT)-Ub was obtained. Optimized crystals of PL ${ }^{\text {pro }}$ (C111S)-Ub were subsequently obtained within two days under the condition: $22 \% \mathrm{w} / \mathrm{v}$ PEG 4000, $15 \% \mathrm{v} / \mathrm{v}$ 2-propanol, $0.1 \mathrm{~mol} / \mathrm{L}$ tri-sodium citrate $\mathrm{pH} 4.8$, and $10 \%$ glycerol. $2 \mu \mathrm{L}$ of protein and $2.5 \mu \mathrm{L}$ of reservoir were mixed to equilibrate against $500 \mu \mathrm{L}$ reservoir.

Crystals were placed in a nitrogen-gas stream $(100 \mathrm{~K})$. A 3.16- $\AA$ dataset was collected at wavelength $0.91841 \AA$ at beamline 14.2 of BESSY, Berlin (Mueller et al., 2012). The program $X D S$ (Kabsch, 2010) was used to process the diffraction data. The space group was found to be $P_{3}$, with unit-cell parameters $a=b=138.14 \AA, c=$ $57.59 \AA, \gamma=120^{\circ}$. Diffraction data statistics are shown in Table 1 .

\section{Phase determination, model building and refinement}

The structure of the MERS-CoV PL ${ }^{\text {pro }}$ (C111S)-Ub complex was solved by molecular replacement using MOLREP (Vagin \& Teplyakov, 2010). The program selected the MERS-CoV PL ${ }^{\text {pro }}$ (Protein Data Bank (PDB) entry 4P16, Lei et al., 2014) as the first search model. Human ubiquitin (PDB entry: 1UBQ, Vijay-Kumar et al., 1987) was used as the second search model. The model of the complex was inspected and rebuilt using Coot (Emsley et al., 2010), and refined using phenix.refine (Headd et al., 2012). The refinement statistics are shown in Table 1. The final model coordinates and struc-
Table 1. Data collection and refinement statistics

\begin{tabular}{|c|c|}
\hline \multicolumn{2}{|c|}{ MERS-CoV PL ${ }^{\text {pro }}(\mathrm{C} 111 \mathrm{~S})-\mathrm{Ub}$} \\
\hline \multicolumn{2}{|c|}{ Data collection statistics } \\
\hline Space group & $\mathrm{P}_{3}$ \\
\hline \multirow[t]{2}{*}{ Unit-cell dimensions $\left(\AA{ }^{\circ}{ }^{\circ}\right)$} & $a=b=138.14, c=57.59$ \\
\hline & $\mathrm{V}=120$ \\
\hline Wavelength $(\AA)$ & 0.91841 \\
\hline$V_{\mathrm{m}}\left(\AA^{3} / \mathrm{Da}\right)$ & 3.59 \\
\hline Solvent content (\%) & 65.8 \\
\hline Resolution range $(\AA)$ & $45.22-3.16(3.33-3.16)$ \\
\hline Number of unique reflections & $10955(1577)$ \\
\hline$R_{\text {pim }}{ }^{1}$ & $0.050(0.413)$ \\
\hline $\operatorname{CC}(1 / 2)$ & $0.997(0.708)$ \\
\hline Completeness (\%) & $99.9(100)$ \\
\hline 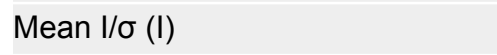 & $12.8(2.0)$ \\
\hline Multiplicity & $7.3(7.5)$ \\
\hline \multicolumn{2}{|l|}{ Refinement statistics } \\
\hline$R_{\text {cryst }}(\%)^{2}$ & 21.1 \\
\hline$R_{\text {free }}(\%)^{2}$ & 25.5 \\
\hline \multicolumn{2}{|l|}{ No. of atoms } \\
\hline Protein & 3054 \\
\hline Ion & 1 \\
\hline Ligand & 24 \\
\hline Clashscore $^{2}$ & 17 \\
\hline r.m.s.deviation in bond lengths $(\AA)$ & 0.012 \\
\hline r.m.s.deviation in bond angles $\left({ }^{\circ}\right)$ & 1.945 \\
\hline Average $B$-factor for all atoms $\left(\AA^{2}\right)$ & 108 \\
\hline Average $B$-factor for $\mathrm{PL}^{\text {pro }}\left(\AA^{2}\right)$ & 95 \\
\hline Average $B$-factor for $U b\left(\AA^{2}\right)$ & 145 \\
\hline
\end{tabular}

Residues in favored regions (\%) $\quad 95.30$

Residues in allowed regions (\%) $\quad 4.44$

Residues in outlier regions (\%) $\quad 0.26$

Note: ${ }^{1}$ Rpim (Weiss \& Hilgenfeld, 1997). ${ }^{2} R_{\text {cryst }}=\sum_{\text {hkl }}$ $\left|\mathrm{F}_{\mathrm{o}}(\mathrm{hkl})-\mathrm{F}_{\mathrm{c}}(\mathrm{hkl})\right| / \Sigma_{\text {hkl }} \mathrm{F}_{\mathrm{o}}(\mathrm{hkl}) . R_{\text {free }}$ was calculated for a test set of reflections $(4.9 \%)$ omitted from the refinement.

${ }^{3} \mathrm{Cl}$-ash-score is defined as the number of clashes calculated for the model per 1000 atoms (including hydrogens) of the model (Chen et al., 2010).

ture factors have been deposited in the PDB database with the code 4WUR. Figures (except for the supplemental figure) have been prepared using Pymol (Schrödinger; http://www.pymol.org/). 
Kinetic assays of purified $\mathrm{PL}^{\text {pro }} \mathrm{S}$

All enzymatic assays were performed using a 96-well microtiter plate and the reaction buffer $20 \mathrm{mmol} / \mathrm{L}$ Tris$\mathrm{HCl}, 150 \mathrm{mmol} / \mathrm{L} \mathrm{NaCl}, \mathrm{pH} 7.9,2 \mathrm{mmol} / \mathrm{L}$ dithiothreitol (DTT). The fluorogenic substrates Cbz-Arg-Leu-ArgGly-Gly-7-amino-4-methylcoumarin (Z-RLRGG-AMC) (Bachem), Z-LRGG-AMC (BostonBiochem), and ubiquitin-AMC (Ub-AMC) (BostonBiochem) were used. The fluorescence of free AMC with different concentrations $(5 \mathrm{nmol} / \mathrm{L}-2.5 \mu \mathrm{mol} / \mathrm{L})$ in reaction buffer was measured to generate a calibration curve, in order to convert the change of fluorescence intensity per unit of time, $\Delta(\mathrm{AFU}) / \mathrm{s}$, into the amount of hydrolyzed substrate in $\mu \mathrm{mol} / \mathrm{L} / \mathrm{s}$.

The enzymatic cleavage reactions were run with an Flx800 fluorescence spectrophotometer (BioTek), to measure the increased fluorescence signal $\left(\lambda_{\mathrm{ex}}: 360 \mathrm{~nm}\right.$; $\lambda_{\mathrm{em}}: 460 \mathrm{~nm}$ ) resulting from AMC release. Reactions were initiated by addition of the proteases to the reaction system. The peptide-hydrolysis kinetic assays were performed with the following conditions: $1 \mu \mathrm{mol} / \mathrm{L}$ MERSCoV PL ${ }^{\text {pro }}$ variant (D164E, D164A, D165E, F269Y), or $10 \mu \mathrm{mol} / \mathrm{L}$ D165A, or $0.1 \mu \mathrm{mol} / \mathrm{L}$ SARS-CoV PL ${ }^{\text {pro }}$, with different concentrations $(10,20,40,80,100 \mu \mathrm{mol} / \mathrm{L})$ of Z-RLRGG-AMC or Z-LRGG-AMC in a final volume of $100 \mu \mathrm{L}$ at $25^{\circ} \mathrm{C}$. The kinetic curves for the proteases and their variants with the substrates Z-RLRGG-AMC or ZLRGG-AMC were linear and the initial velocities also increased linearly with substrate concentration. No saturation was observed. Therefore, the data were fitted to the equation $\mathrm{v} /[\mathrm{E}]_{\text {tot. }}=k_{\text {app }}[\mathrm{S}]$, where $k_{\text {app }}$ approximates $k_{c a} / K_{M}$, as described previously (Barretto et al., 2005; Wojdyla et al., 2010).

The deubiquitinating kinetic assays were performed under the following conditions: $0.1 \mu \mathrm{mol} / \mathrm{L}$ MERS-CoV $\mathrm{PL}^{\mathrm{pro}}$ wild-type or its variants D164E, D164A, D165E, F269Y, or $0.5 \mu \mathrm{mol} / \mathrm{L}$ D165A, or $0.025 \mu \mathrm{mol} / \mathrm{L}$ SARS$\mathrm{CoV} \mathrm{PL}^{\mathrm{pro}}$ were incubated with increasing concentrations $(1,2,4,8 \mu \mathrm{mol} / \mathrm{L})$ of $\mathrm{Ub}-\mathrm{AMC}$ in a final volume of $50 \mu \mathrm{L}$, at $25^{\circ} \mathrm{C}$. Although $\mathrm{PL}^{\text {pro }}$ actually cleaves the isopeptide bond between the carboxyl group of the C-terminal Gly in Ub and the $\varepsilon$-amino group of Lys in ubiquitinated targets in vivo, we used the hydrolysis of $\mathrm{Ub}-$ AMC here to test the deubiquitinating activity in vitro. The kinetic curves of proteases and variants with the substrate Ub-AMC were hyperbolic and the initial velocities were not linear over the concentration of substrate. However, saturation was still not observed within a reasonable time. Only when the ratio of protein to substrate was 1:1 or larger, were we able to achieve saturation within a limited time (data not shown). As the initial velocities did not increase in a strictly linear fashion with substrate concentration, application of the equation $\mathrm{v} /[\mathrm{E}]_{\text {tot. }}=k_{\text {app }}[\mathrm{S}]$ to mimic $k_{c a t} / K_{M}$ would lead to large standard errors. We were however able to fit the data to the Michaelis-Menten equation using the GraphPad Prism program (GraphPad Software), even though saturation could not be reached (Supplemental Figure 1). All assays were performed in duplicates.

\section{RESULTS}

Overall structure of MERS-CoV PL ${ }^{\text {pro }}$ in complex with human ubiquitin

The substrate-binding site of MERS-CoV PL ${ }^{\text {pro }}$ features significant differences from those of the corresponding SARS-CoV enzyme and human ubiquitin-specific proteases (USPs, such as, USP14) (Hu et al., 2005; Chou et al., 2014; Ratia et al., 2014). It is therefore of interest to determine the crystal structure of the complex between MERS-CoV PL ${ }^{\text {pro }}$ and its substrate, human ubiquitin. Hence, we crystallized the ubiquitin ( $\mathrm{Ub}$ ) complex of a MERS-CoV PL ${ }^{\text {pro }}$ variant that had the active-site Cys 111 replaced by serine $(\mathrm{C} 111 \mathrm{~S})$ and determined the structure at $3.16 \AA$ (Figure $1 \mathrm{~A}$ ).

There is one PL ${ }^{\mathrm{pro}}(\mathrm{C} 111 \mathrm{~S})-\mathrm{Ub}$ complex per asymmetric unit. Using the PDBePISA server (Krissinel \& Henrick, 2007), the total interface region of MERS-CoV $\mathrm{PL}^{\text {pro }}$ (C111S)-Ub was determined as $813 \AA^{2}$, close to the $915 \AA^{2}$ interface of SARS-CoV PL ${ }^{\text {pro }}$-Ubal (ubiquitin aldehyde; PDB entry: 4MM3, Ratia et al., 2014) and the $999 \AA^{2}$ of SARS-CoV PL ${ }^{\text {pro }}$ (C112S)-Ub (PDB: 4M0W, Chou et al., 2014), but less than the $1503 \AA^{2}$ observed for USP14-Ubal (PDB: 2AYO, Hu et al., 2005). The overall structure of MERS-CoV PL ${ }^{\text {pro }}(\mathrm{C} 111 \mathrm{~S})$ is very similar to that of the substrate-free $\mathrm{PL}^{\text {pro }}$ (Lei et al., 2014), with a root-mean-square deviation (RMSD) of $0.91 \AA$ for corresponding $\mathrm{C} \alpha$ atoms between these two structures. Nevertheless, two differences are immediately visible: 1 ), the zinc-finger motif has moved and closed in onto the $\mathrm{Ub}$, compared to the free $\mathrm{PL}^{\mathrm{pro}}$. The zinc ion position has shifted by about $4 \AA$ and the largest deviation between the two structures is $\sim 6 \AA$ for Cys 228 of the zinc-finger region (Figure 1B); 2) the mobile loop ${ }^{271}$ GIETAVG ${ }^{277}$, also named "BL2 loop", is defined by clear main-chain electron density (Figure 1C). This loop is disordered in substrate-free PL $^{\text {pro }}$ (Lei et al., 2014; Bailey-Elkin et al., 2014). Compared to MERS-CoV PL ${ }^{\text {pro }}$, the BL2 loop ${ }^{267} \mathrm{GNYQCG}^{272}$ is shorter by one residue in SARS-CoV $\mathrm{PL}^{\text {pro }}$.

At the time when we deposited in the PDB the coordinates for the crystal structure of the non-covalent complex MERS-CoV (C111S)-Ub (PDB: 4WUR), Bailey-Elkin et al. (2014) described two crystal structures for a covalent complex MERS-CoV PL ${ }^{\text {pro }}-\mathrm{Ub}$, in which an alkyl bromide group introduced at the C-terminus of $\mathrm{Ub}$ had formed a thioether with the active-site Cys111 of MERS-CoV PL ${ }^{\text {pro }}$. These structures were in 


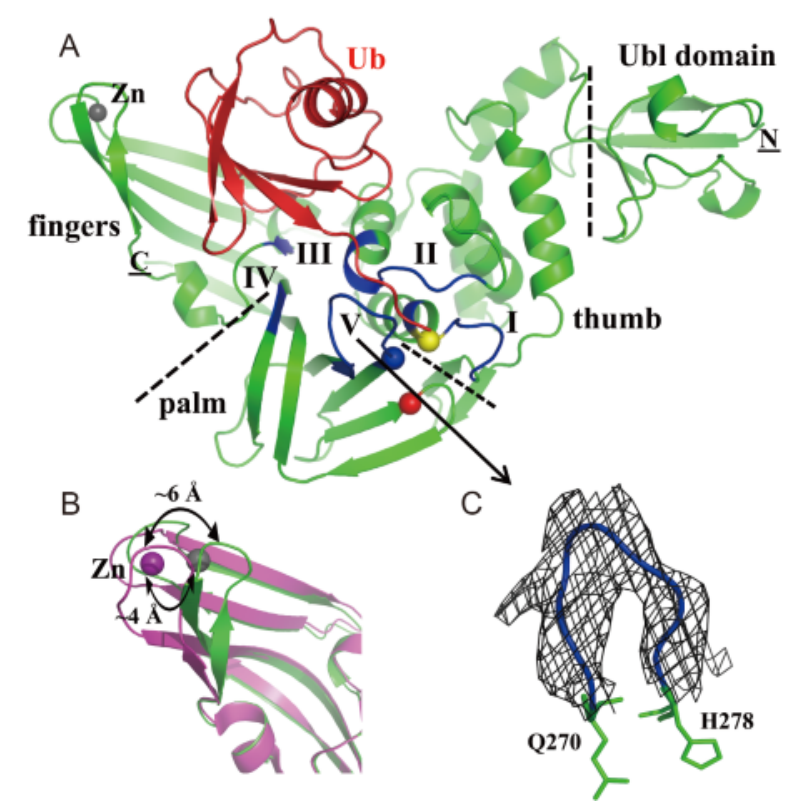

Figure 1. Structure of MERS-CoV $\mathrm{PL}^{\text {pro }}$ in complex with human ubiquitin (Ub). (A) Cartoon view of the overall complex structure. $\mathrm{PL}^{\text {pro }}$ is shown in green and $\mathrm{Ub}$ in red. The $\mathrm{N}$ and $\mathrm{C}$ termini are marked by underlined letters, and the $\mathrm{PL}^{\text {pro }}$ subdomains are divided by black dashed lines. The ubiquitin-like (Ubl) domain, thumb, palm, and fingers subdomains are indicated. The catalytic triad Cys111-His278-Asp293 is shown by spheres (yellow, blue, and red; Cys was replaced by Ser in this study). The zinc atom is displayed as a gray sphere. Five $\mathrm{PL}^{\text {pro }}$ regions interacting with $\mathrm{Ub}$ are colored dark blue and labeled with Roman numbers (I-V). (B) Superposition of the fingers domain of Ub-bound MERS-CoV $\mathrm{PL}^{\text {pro }}$ and the substrate-free enzyme (PDB: 4P16; Lei et al., 2014). The zinc atom is shifted by $\sim 4 \AA$, and the largest difference is $\sim 6 \AA$. (C) The BL2 loop (blue; ${ }^{271} \mathrm{GI}$ $\mathrm{ETAVG}^{277}$ ) of $\mathrm{PL}^{\text {pro }}$. A $2 \mathrm{~F}_{\mathrm{o}}-\mathrm{F}_{\mathrm{c}}$ electron density (gray; 1.0 $\sigma)$ is displayed. The side-chains of residues in this loop are only partly defined by electron density.

space groups $\mathrm{P}_{5} 22$ and $\mathrm{P}_{3}$ and were named "closed" and "open" PL ${ }^{\text {pro }}$-Ub complexes (PDB: 4RF0 and 4RF1), respectively (Bailey-Elkin et al., 2014). Even though the zinc-finger motif of $\mathrm{PL}^{\text {pro }}$ in the former complex (space group $\mathrm{P}_{5} 22$ ) is closer to the $\mathrm{Ub}$ than in the $\mathrm{P}_{3}$ complex, both the "closed" and "open" complex show almost the same interactions between PL ${ }^{\text {pro }}$ and Ub (Bailey-Elkin et al., 2014). The differences may be caused by crystal packing. Our non-covalent complex is similar to the "open" form of the covalent PL ${ }^{\text {pro }}$-Ub complex (RMSD = $0.58 \AA$ for the $\mathrm{PL}^{\text {pro }}$ and $0.85 \AA$ for $\mathrm{Ub}$, based on all $\mathrm{C} \alpha$ atoms).

All parts of the $\mathrm{PL}^{\text {pro }}$ except for the ubiquitin-like (Ubl) domain interact with Ub. Most interactions in-
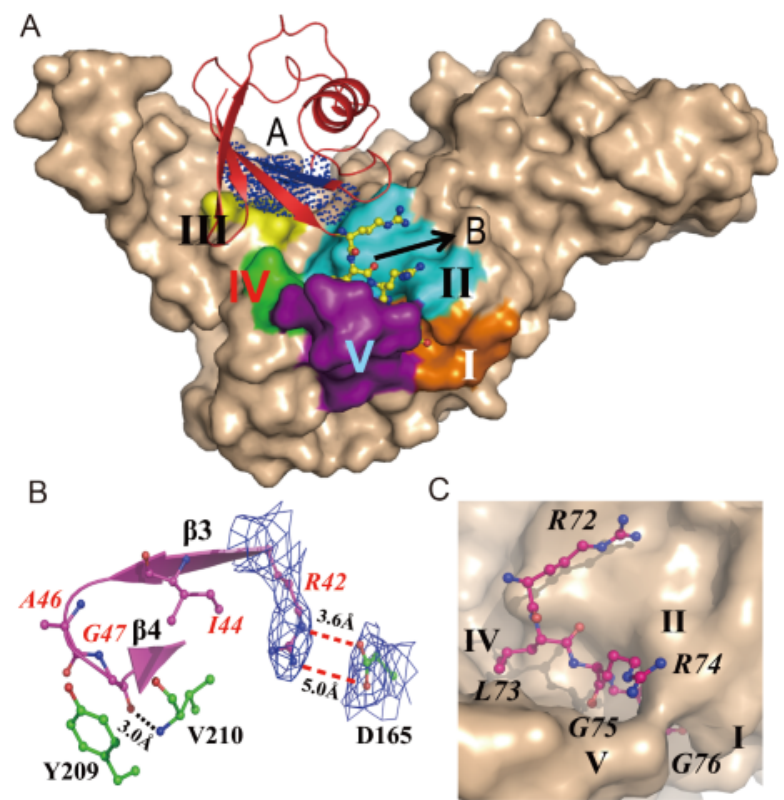

Figure 2. Interactions between MERS-CoV $\mathrm{PL}^{\text {pro }}$ and Ub. (A) Five regions of $\mathrm{PL}^{\text {pro }}$ bind to two areas of $\mathrm{Ub}$. The surface of $\mathrm{PL}^{\text {pro }}$ is shown in wheat color. The five interacting regions are shown in brown, cyan, yellow, green, and purple, and they are also labeled by Roman numbers. The two interacting areas of Ub are marked " $A$ " and "B". Region A is highlighted by dark blue dots, and region $\mathrm{B}$ (the $R L R G G$ sequence; $U b$ residues are in italics) is depicted in the ball-and-stick style. (B) Region $A$ of $\mathrm{Ub}$ interacting with $\mathrm{PL}^{\text {pro }}$. A cartoon view of region $A$ is shown in purple, and $\beta 3$ and $\beta 4$ are labeled. Residues of Ub (purple) and $\mathrm{PL}^{\text {pro }}$ (green) are displayed in the ball-and-stick style, and labeled in red and black, respectively. The $2 \mathrm{~F}_{\mathrm{o}}-\mathrm{F}_{\mathrm{c}}$ electron density (blue; $1.0 \sigma$ ) of the side-chains of Arg42 and Asp165 is displayed. Hydrogen bonds are indicated by black dashed lines, and the salt-bridge between $R 42$ and D165 is depicted by two red dashed lines. (C) The RLRGG binding site of $\mathrm{PL}^{\text {pro }}$. The P5-P1 residues are shown in the ball-and-stick style. Regions I, II, IV, and V that interact with the P5-P1 residues are labeled.

volve five surface regions of the $\mathrm{PL}^{\text {pro }}$ and two regions of $\mathrm{Ub}$ (Figure 2A). These five regions of the $\mathrm{PL}^{\text {pro }}$ are labelled by Roman numbers: I, Leu106-Tyr112; II, A la 162-Arg168; I I I, Cys $208-\mathrm{Va} 1210$; IV, Gly248-Pro250; V, Phe269-Tyr279. I and II are situated in the thumb domain; III is in the fingers domain; and IV and $\mathrm{V}$ are in the palm domain (Figures $1 \mathrm{~A}$ and 2A). In the following and in the figure labels, residues of $\mathrm{Ub}$ are indicated in italics to distinguish them from residues of $\mathrm{PL}^{\text {pro }}$. The two interacting regions of $\mathrm{Ub}$ are: A, Arg42-Gln49; B, Arg72-Gly76 (Figure 2A). Region A of $\mathrm{Ub}$ consists of $\beta 3, \beta 4$, and the loop between $\beta 3$ and 
$\beta 4$ (Figure 2B). Regions II and III of $\mathrm{PL}^{\text {pro }}$ interact with region $\mathrm{A}$ of $\mathrm{Ub}$. Region $\mathrm{B}$ comprises the five $\mathrm{C}$-terminal residues, $R L R G G$, and is in contact with regions $\mathrm{I}, \mathrm{II}, \mathrm{IV}$, and $\mathrm{V}$ of $\mathrm{PL}^{\text {pro }}$ (Figure 2C). The C-terminal RLRGG motif contributes the majority of the interactions with the $\mathrm{PL}^{\text {pro }}$; the buried surface between $\mathrm{Ub}$ region $\mathrm{B}$ and $\mathrm{PL}^{\text {pro }}$ is $477 \AA^{2}$ (out of a total of $813 \AA^{2}$ ). In order to make these interactions clear, we describe here in some detail the contacts between the $\mathrm{PL}^{\text {pro }}$ and regions $\mathrm{A}$ and $\mathrm{B}$ of $\mathrm{Ub}$.

\section{Interactions of MERS-CoV PL ${ }^{\text {pro }}$ (C111S) with Ub region $\mathrm{A}$}

Region A (Arg42-Gln49) of Ub inserts into the space between the thumb and fingers domains of $\mathrm{PL}^{\mathrm{pro}}$ (Figures 2A-B). Residues Ile44, Ala46, and Gly47 engage in hydrophobic interactions with Tyr209 and Val210 of region III of $\mathrm{PL}^{\text {pro }}$ (Figure 2B). The hydrophobic patch (Ile44, Ala46, and Gly47) is a common interaction region utilized by Ub-binding proteins (Dikic et al., 2009). In particular, the interaction of Ile 44 with Val210 of $\mathrm{PL}^{\mathrm{pro}}$ is important for the deubiquitinase (DUB) but not for the protease activity. The variant V210R shows dramatically reduced DUB activity, as demonstrated by Bailey-Elkin et al. (2014) (according to the numbering scheme of these authors, V210 is V1691).

A salt-bridge exists between region $\mathrm{A}$ of $\mathrm{Ub}$ and the $\mathrm{PL}^{\text {pro }}$ (Figure 2B), namely between the side-chains of Arg42 and Asp165 (region II in PL ${ }^{\text {pro }}$ ). In addition, a hydrogen bond is formed between the main-chain $\mathrm{O}$ atom of Gly47 and the main-chain amide of Val210 (region III). In the SARS-CoV PL ${ }^{\text {pro }}$ (C112S)-Ub complex (Chou et al., 2014), $\operatorname{Arg} 42$ forms a salt-bridge with the negatively charged Glu168, a residue which is replaced by the positively charged Arg168 in MERS-CoV PL ${ }^{\text {pro }}$. Consequently, the same salt-bridge cannot be formed in the
MERS-CoV PL ${ }^{\text {pro }}$ (C111S) -Ub complex. Instead, MERS-CoV PL ${ }^{\text {pro }}$ has Asp165 interacting with Arg42. In our deubiquitinating (DUB) kinetic assays, the D165A variant shows a dramatically reduced DUB activity; the $k_{c a t} / K_{M}$ is about 78 -fold decreased compared to that of the wild-type (Table 2). The $K_{M}$ value of the D165A variant is about 4-fold higher than that of the wild-type MERS-CoV PL ${ }^{\text {pro }}$, suggesting that this amino-acid replacement reduces the Ub binding affinity. Meanwhile, the D165E amino-acid replacement shows a catalytic efficiency comparable to wild-type towards Ub-7-amino-4methylcoumarin (Ub-AMC) (Table 2). However, we noticed that the $K_{M}$ value of D165E for the DUB activity is about 2-fold larger than for the WT enzyme. Although Glu165 can mimic Asp165 here, we propose that the longer side-chain of Glu may fit less perfectly compared to Asp. These results indicate that the salt-bridge between Asp165 and Arg42 could be important for the $\mathrm{PL}^{\mathrm{pro}}$, $\mathrm{D}$ DUB activity, in addition to the interaction between Asp165 and the P4-amide group (see below).

\section{Interactions of MERS-CoV PL ${ }^{\text {pro }}$ (C111S) with Ub region $B$}

Region B of Ub comprises the five C-terminal residues, $R L R G G$. These five residues bind to the narrow activesite channel between the thumb and palm domains of the PL $^{\text {pro }}$ (Figure 2C). They mainly interact with regions I, II, $\mathrm{IV}$, and $\mathrm{V}$ of the protease. Residues $R L R G G$ are compatible with the PL ${ }^{\text {pro }}$ cleavage motif, $(\mathrm{R} / \mathrm{K})(\mathrm{L} / \mathrm{I}) \mathrm{XGG}$ (P5-P1), in the MERS-CoV polyproteins; their interactions with the $\mathrm{PL}^{\mathrm{pro}}$ are discussed here in terms of subsites $\mathrm{S} 1$ to $\mathrm{S} 5$.

S1 and S2 subsites. Pro163 (region II of $\mathrm{PL}^{\text {pro }}$ ) and the side-chains of Asn109 and Tyr112 (located in region I)

Table 2. Kinetic parameters of MERS-CoV PL ${ }^{\text {pro }}$ and SARS-CoV PL ${ }^{\text {pro }}$

\begin{tabular}{|c|c|c|c|c|c|}
\hline & \multicolumn{2}{|c|}{ Z-RLRGG-AMC Z-LRGG-AMC } & \multicolumn{3}{|c|}{ Ub-AMC } \\
\hline & \multicolumn{2}{|c|}{$k_{\text {app }}\left(\mu \mathrm{mol}^{-1} \min ^{-1}\right)^{*}$} & $K_{M}(\mu \mathrm{mol} / \mathrm{L})$ & $k_{\text {cat }}\left(\min ^{-1}\right)$ & $k_{\text {cat }} / K_{M}\left(\mu \mathrm{mol} / \mathrm{L}^{-1} \mathrm{~min}^{-1}\right)$ \\
\hline \multicolumn{6}{|c|}{ MERS-CoV PL pro } \\
\hline$W T^{\#, 1}$ & $(1.3 \pm 0.1) \times 10^{-3}$ & $(1.0 \pm 0.01) \times 10^{-3}$ & $6.1 \pm 0.7$ & $8.4 \pm 0.7$ & $1.4 \pm 0.2$ \\
\hline F269Y & $(2.0 \pm 0.1) \times 10^{-3}$ & $(1.4 \pm 0.2) \times 10^{-3}$ & $8.5 \pm 0.1$ & $22.4 \pm 1.5$ & $2.6 \pm 0.2$ \\
\hline D165E & $(1.1 \pm 0.2) \times 10^{-3}$ & $(8.4 \pm 1.1) \times 10^{-4}$ & $15.9 \pm 2.7$ & $27.7 \pm 1.2$ & $1.7 \pm 0.3$ \\
\hline D165A & $(8.2 \pm 1.6) \times 10^{-5}$ & $(1.0 \pm 0.2) \times 10^{-4}$ & $23.9 \pm 6.2$ & $0.42 \pm 0.04$ & $0.018 \pm 0.005$ \\
\hline D164E & $(1.5 \pm 0.1) \times 10^{-3}$ & $(8.6 \pm 1.8) \times 10^{-4}$ & $15.7 \pm 4.4$ & $15.3 \pm 3.3$ & $1.0 \pm 0.3$ \\
\hline D164A & $(2.9 \pm 0.1) \times 10^{-4}$ & $(6.5 \pm 0.8) \times 10^{-4}$ & $6.5 \pm 0.6$ & $2.5 \pm 0.01$ & $0.40 \pm 0.04$ \\
\hline$W T^{\#, 2}$ & $(3.0 \pm 0.1) \times 10^{-3}$ & & $14.3 \pm 2.0$ & $18.8 \pm 1.2$ & $1.3 \pm 0.2$ \\
\hline \multicolumn{6}{|c|}{ SARS-CoV PL pro } \\
\hline$W T^{\#, 1}$ & $0.23 \pm 0.04$ & $(3.5 \pm 0.5) \times 10^{-2}$ & $57.2 \pm 10.9$ & $197.4 \pm 28.0$ & $3.5 \pm 0.8$ \\
\hline $\mathrm{WT}^{\#, 2}$ & $0.3 \pm 0.1$ & & $50.6 \pm 7.4$ & $75.9 \pm 8.1$ & $1.5 \pm 0.3$ \\
\hline
\end{tabular}

Note: ${ }^{\#}$ : Wild type; * $: k_{\text {app }}$ is an approximation to $k_{c a t} / K_{M} ;{ }^{1}:$ Our results (this work); ${ }^{2}$ : Baez-Santos et al., 2014b. 
form a space-restricted S1 site to accommodate Gly 76 (P1). The carbonyl oxygen atom of Gly277 (region V) accepts a hydrogen bond from the amide of Gly76 (Figure 3).

The side-chains of Tyr112 (region I) and Phe269, Val276 as well as Tyr279 (region V), and Gly277 form a restricted space for Gly 75 (P2). Two hydrogen bonds link the main-chain at Asp164 of the $\mathrm{PL}^{\text {pro }}$ and Gly75 (Figure 3). The S1 and S2 sites of the protease are too small to accommodate any other residue but glycine.

S3 subsite. The main-chain O atom of $\operatorname{Arg} 74$ (P3) accepts a hydrogen bond from the Gly277 amide. In the complexes SARS-CoV PL ${ }^{\text {pro }}(\mathrm{C} 112 \mathrm{~S})-\mathrm{Ub}$ or SARS-CoV $\mathrm{PL}^{\text {pro }}$-Ubal, the main chain at $\mathrm{Arg} 74$ forms two hydrogen bonds (Chou et al., 2014; Ratia et al., 2014), namely with the amide of Gly272 and with the hydroxyl group of Tyr265. The former hydrogen bond is conserved in the MERS-CoV PL ${ }^{\text {pro }}$ (C111S)-Ub complex, but the latter is not. Tyr265 of SARS-CoV is replaced by Phe 269 in MERS-CoV, which lacks the ability to form a hydrogen bond with the main-chain amide of $\mathrm{Arg} 74$. In agreement with this difference, the DUB activity of SARS-CoV

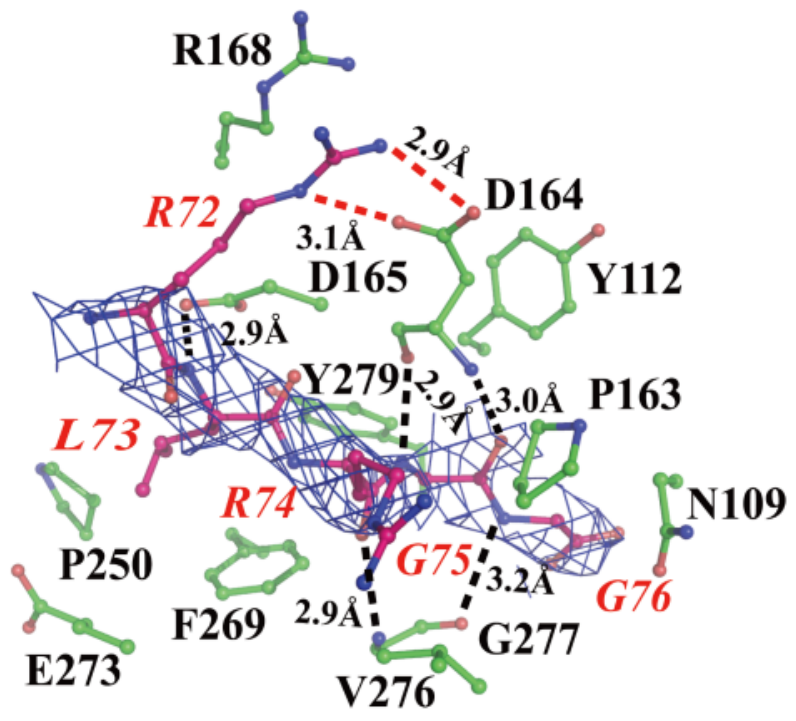

Figure 3. Details of the interactions between the C-terminal RLRGG of Ub and MERS-CoV PL ${ }^{\text {pro }}$. RLRGG residues are shown in purple in the ball-and-stick style, and they are labeled in red. For clarity, the $2 \mathrm{~F}_{\mathrm{o}}-\mathrm{F}_{\mathrm{c}}$ electron density (blue; $1.0 \sigma$ ) of the RLRGG main chain is shown. $\mathrm{PL}^{\text {pro }}$ residues are displayed in green in the balland-stick style, and they are labeled in black. Hydrogen bonds are displayed as black dashed lines, and the salt-bridge between $R 72$ and D164 is depicted as two red dashed lines.
$\mathrm{PL}^{\text {pro }}$ is 2.5-fold higher than that of MERS-CoV PL ${ }^{\text {pro }}$ in our kinetic assay; furthermore, the MERS-CoV PL ${ }^{\text {pro }}$ F269Y amino-acid replacement leads to enhancements by about 1.5-, 1.4-, and 1.9-fold of the hydrolytic activities towards carbobenzoxy-Arg-Leu-Arg-Gly-Gly-7amino-4-methylcoumarin (Z-RLRGG-AMC), Z-LRGG$\mathrm{AMC}$, and Ub-AMC, respectively (Table 2).

The side-chain of $\mathrm{Arg} 74$ is exposed to the solvent in our MERS-CoV PL ${ }^{\text {pro }}$ (C111S)-Ub complex. This sidechain shows remarkable variability in the interactions it makes in the different complexes. In the "open" but not in the "closed" covalent MERS-CoV PL ${ }^{\text {pro }}$-Ub complex (Bailey-Elkin et al., 2014), it donates a hydrogen bond to the main-chain carbonyl oxygen of Thr1755 of the BL2 loop (corresponding to Thr274 in our numbering scheme). In SARS-CoV PL ${ }^{\text {pro }}$-Ubal (Ratia et al., 2014) but not in the SARS-CoV PL ${ }^{\text {pro }}$ (C112S)-Ub complex (Chou et al., 2014), the side-chain of Arg74 forms a hydrogen bond with the main-chain carbonyl oxygen of Gln270. Instead, $\operatorname{Arg} 74$ is involved in a relatively weak salt-bridge with Glu162 in the SARS-CoV PL ${ }^{\text {pro }}$ (C112S)-Ub complex (Chou et al., 2014). None of these interactions exist in our MERS-CoV PL ${ }^{\text {pro }}-\mathrm{Ub}$ complex.

S4 subsite. The main-chain amide of Leu73 (P4) donates a hydrogen bond to the side-chain of Asp165 (region II) (Figure 3). This hydrogen bond is conserved in all the complexes compared here. In our peptide-cleavage assay, the D165A variant shows about 16 -fold and 10 -fold lower activities towards substrates Z-RLRGG-AMC and Z-LRGG-AMC, respectively, indicating that Asp165 is not only important for interacting with $\mathrm{Arg} 42$ of $\mathrm{Ub}$ (see above).

The side-chain of Leu73 is embedded in a hydrophobic pocket which is formed by the $C \beta$ atom of Asp165, the side-chain of Pro250 (region IV), Phe269, as well as the $\mathrm{C} \beta$ and $\mathrm{C} \gamma$ atoms of Glu273 (region V). Asp165 and Pro250 are conserved in SARS-CoV PL ${ }^{\text {pro }}$ (Asp165 and Pro249). Phe269 is replaced by Tyr265, and Glu273, situated in the BL2 loop, is replaced by Tyr269. However, the side-chains of all these non-conserved residues possess the ability to provide a hydrophobic environment to accommodate Leu73.

S5 subsite. The side-chain of $\operatorname{Arg} 72$ (P5) is located between the $\mathrm{PL}^{\text {pro }}$ thumb domain and region A of ubiquitin. It forms a salt-bridge with the side-chain of Asp164 in MERS-CoV PL ${ }^{\text {pro }}$. In addition, the guanidinum group of $\operatorname{Arg} 72$ may be involved in a $\pi-\pi$ interaction with that of Arg168 (Figure 3). These interactions have also been described for the covalent complex of MERS-CoV PL ${ }^{\text {pro }}$ with Ub (Bailey-Elkin et al., 2014). Arg72 is not subject to strict space limitations; accordingly, this residue displays different binding patterns in the two SARS-CoV 
$\mathrm{PL}^{\text {pro }}$-Ub complexes. In the covalent SARS-CoV PL ${ }^{\text {pro }}$ Ubal complex (Ratia et al., 2014), Arg72 forms a saltbridge with Glu168. In the non-covalent SARS-CoV $\mathrm{PL}^{\text {pro }}$ (C112S)-Ub complex (Chou et al., 2014), Arg72 is exposed to the solvent and does not interact with Glu168 (Arg42 instead forms a salt-bridge with Glu168, as mentioned above). In our kinetic assay, the D164A variant of MERS-CoV PL ${ }^{\text {pro }}$ displays a $\sim 4.5$-fold and a $\sim 3.5$-fold reduced activity, respectively, for Z-RLRGG-AMC and Ub-AMC (Table 2). For Z-LRGG-AMC, the activity is decreased just a little (by about $\sim 1.5$-fold), because there is no P5-Arg in this substrate (Table 2). These data demonstrate that Asp164 is important for the interaction with $\operatorname{Arg} 72$.

In summary, the main-chain heteroatoms of P5-P1 form a hydrogen-bonding network with $\mathrm{PL}^{\mathrm{pro}}$. The binding characteristics of $\mathrm{P} 1, \mathrm{P} 2$, and $\mathrm{P} 4$ are conserved in all MERS-CoV and SARS-CoV PL ${ }^{\text {pro }}$-Ub complexes. However, P3-Arg and P5-Arg assume binding patterns that differ between the various MERS-CoV and SARS$\mathrm{CoV} \mathrm{PL}^{\mathrm{pro}}-\mathrm{Ub}$ complexes.

\section{DISCUSSION}

Viral proteins are likely to possess multiple functions, as exemplified by non-structural protein 1 (NS1) of influenza A viruses (Hale et al., 2008), the nucleocapsid (N) protein of coronaviruses (Chang et al., 2014), or the Nsp14 exonuclease-guanyl-7-methyltransferase of coronaviruses (Chen et al., 2009). Exhibiting DUB and proteolytic activities, the papain-like proteases of coronaviruses are no exception here. Although the overall folds are conserved, the enzyme activity and substrate-binding modes of CoV PL ${ }^{\text {pro }}$ s differ in detail. Therefore, no coronavirus PL ${ }^{\text {pro }}$ can be considered a general model for all its homologues (Baez-Santos et al., 2014b).

\section{MERS-CoV PL ${ }^{\text {pro }}$ is not optimized for catalytic efficiency}

We have previously noticed that the oxyanion hole of the MERS-CoV PL ${ }^{\text {pro }}$ appears to be deficient (Lei et al., 2014; also see the discussion below). Similarly, the recognition of ubiquitin by the enzyme appears to be suboptimal. Thus, the main-chain amide of the P3-Arg residue has no hydrogen-bonding partner on the MERSCoV PL ${ }^{\text {pro }}$, because the near-by side-chain of Phe269 is incapable of accepting an H-bond. The corresponding residue is Tyr265 in the SARS-CoV PL ${ }^{\text {pro }}$, which is perfectly positioned to accept the hydrogen bond from the P3-amide. Indeed, when we replaced Phe269 by Tyr in MERS-CoV PL ${ }^{\text {pro }}$, the DUB activity of the enzyme increased by a factor of almost 2 and the peptidolytic activity by $\sim 1.5$. The evolution of viral enzymes is not neces- sarily driven by optimization of catalytic efficiency. This is particularly true for viral proteases that have to ensure the availability of non-structural proteins in the correct temporal order when they cleave them out of the viral polyproteins; in fact, too rapid a polyprotein processing might be counterproductive. On the other hand, a more efficient DUB activity should help the virus in counteracting the innate immune response of the host cell. As the binding of the P5-P1 residues of ubiquitin and of the polyprotein cleavage sites obviously influences both the DUB and proteolytic activities of the $\mathrm{PL}^{\mathrm{pro}}$, the suboptimal catalytic efficiency that we observe may be a consequence of a compromise between the requirements of the two activities.

With regard to the oxyanion hole, we previously proposed that the backbone amide of Asn 109 (located in a $\beta$-turn connecting $\beta 7$ and $\alpha 4$, Figure 4 ) may contribute to the stabilization of the oxyanion intermediate in $\mathrm{PL}^{\text {pro }}$ catalysis, along with the main-chain amide of the active site Cys111, although this may require a slight rearrangement of this $\beta$-turn (Lei et al., 2014). In our non-covalent MERS-CoV PL ${ }^{\text {pro }}$-Ub complex, we do not see any rearrangement of the $\beta$-turn. More or less in agreement with our suggestion, Bailey-Elkin et al. (2014) proposed

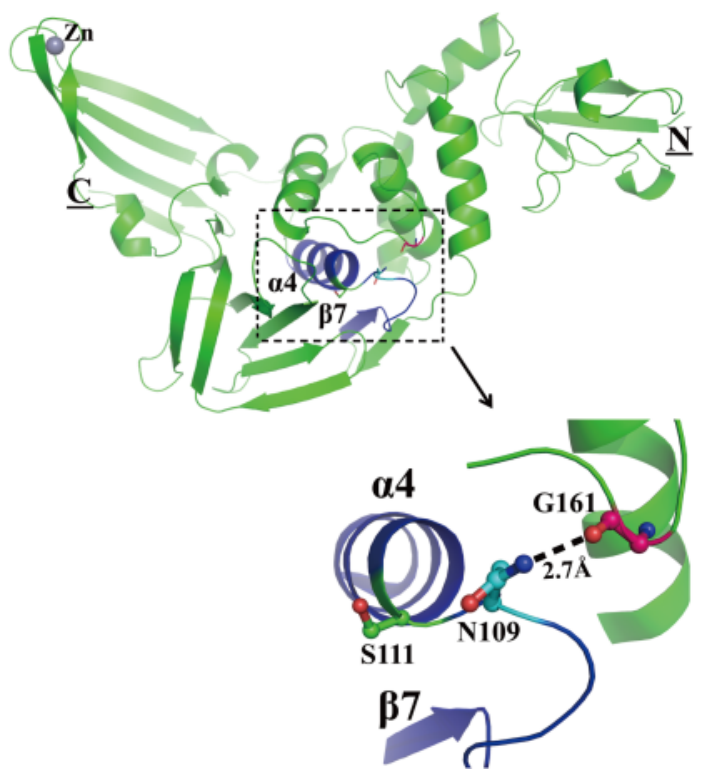

Figure 4. The side-chain of Asn109 is unlikely to contribute to the oxyanion hole of MERS-CoV PL ${ }^{\text {pro }}$. $\mathrm{PL}^{\text {pro }}$ is shown in green in cartoon view. $\mathrm{N}$ and $\mathrm{C}$ termini are marked by underlined letters. Strand $\beta 7$, helix $\alpha 4$, and the loop between them are colored in blue. Asn109 and Gly161 are indicated in ball-and-stick style in cyan and purple, respectively, and they are labeled in black. The hydrogen bond between them is displayed as a black dashed line. The active-site Cys111 (replaced by Ser in this study) is shown in green in ball-and-stick style. 
on the basis of their covalent MERS-CoV PL ${ }^{\text {pro }}-\mathrm{Ub}$ complex structure that the main-chain amides of Asn1590, Asn 1591, and Cys 1592 (corresponding to Asn109, Asn110, and Cys111 in our numbering scheme) form the oxyanion hole. On the other hand, Lee et al. (2015) argued that the side-chain of Asn109 could contribute to the oxyanion hole. They found that the N109A replacement completely abolished enzyme activity. As we reported earlier (Lei et al., 2014), the side-chain amide of Asn 109 makes a strong hydrogen bond with the conserved Gly161 (Figure 4). Any reorientation of the Asn 109 side-chain towards the oxyanion would require a disruption of this strong interaction; this is not very likely. In conclusion, lacking a side-chain in the proper spatial orientation and capable of donating a hydrogen bond to the oxyanion (such as Trp107 in SARS-CoV $\mathrm{PL}^{\mathrm{pro}}$ ), the oxyanion hole of MERS-CoV $\mathrm{PL}^{\text {pro }}$ seems to be deficient.

\section{Unique features of Ub recognition by MERS- CoV PL ${ }^{\text {pro }}$}

Apart from the less than optimum binding of the P3 residue, there are other differences in the way MERSCoV PL ${ }^{\text {pro }}$ and SARS-CoV PL ${ }^{\text {pro }}$ recognize human ubiquitin. The formation of a salt-bridge between $\operatorname{Arg} 72$, the P5 side-chain of Ub, and Asp164 is unique for MERSCoV PL ${ }^{\text {pro }}$. The same interaction exists in the two covalent MERS-CoV PL ${ }^{\text {pro }}$-Ub complexes (Bailey-Elkin et al., 2014). This binding mode is very different from the Glu168 - Arg 72 salt-bridge in the SARS-CoV PL ${ }^{\text {pro }}$-Ubal complex (Ratia et al., 2014). Glu168 is conserved in $\mathrm{HCoV}-\mathrm{NL} 63 \mathrm{PL} 2^{\text {pro }}$ and replaced by Asp in the HCoV229E, HCoV-OC43, and HCoV-HKU1 PL2 ${ }^{\text {pro }}$ s (for sequence alignments, see Barretto et al., 2005; Baez-Santos et al., 2014b), so the same type of interaction is likely to be realized in the $\mathrm{Ub}$ complexes of these enzymes. However, the corresponding residue in MERS-CoV $\mathrm{PL}^{\text {pro }}$ is Arg 168; hence, Asp164 is used instead to bind Arg72. Asp164 is in fact unique in MERS-CoV. It is replaced by Gly in SARS-CoV PL ${ }^{\text {pro }}$ and the PL2 ${ }^{\text {pro }}$ s of HCoV NL63 and HCoV 229E, by Ala in HCoV-OC43 PL2 ${ }^{\text {pro }}$, and by Ser in HCoV-HKU1 PL2 ${ }^{\text {pro }}$. Our kinetic results for the D164A replacement (see Results) emphasize the importance of the unique Asp164 residue.

\section{The role(s) of the ubiquitin-like (Ubl) domain}

In our $\mathrm{PL}^{\text {pro }}(\mathrm{C} 111 \mathrm{~S})-\mathrm{Ub}$ complex, the Ubl domain of $\mathrm{PL}^{\mathrm{pro}}$ shows no interaction with ubiquitin. The relative orientation of the Ubl domain in the substrate-bound $\mathrm{PL}^{\text {pro }}$ is the same as in substrate-free $\mathrm{PL}^{\text {pro }}$. The Ubl domain of MERS-CoV PL ${ }^{\text {pro }}$ is not required for the IFN antagonism activities (Baez-Santos et al., 2014b), but it is required in case of SARS-CoV PL ${ }^{\text {pro }}$ (Frieman et al., 2009). Recently, Mielech et al. (2015) reported that the
Ubl domain of mouse hepatitis virus (MHV) is an important modulator of $\mathrm{PL}^{\text {pro }}$ stability and viral pathogenesis. Although the Ubl domain shows variable effects in different CoVs, the high degree of conservation of the domain throughout the family suggests that it may play a common biological role. One possible function is that the Ubl might be involved in protein-protein interactions. Ubiquitin-like domains are known to function as binding modules in such interactions. For example, the kinase Raf contains a Ubl domain for interaction with human Ras (Fetics et al., 2015). Also, human ubiquitin-specific protease (USP) 7 includes five Ubl (1-5) domains, of which the second is bound by the Herpes simplex virus-1 immediate-early protein ICP0 to counteract the intrinsic antiviral response of the host cell (Pfoh et al., 2015).

\section{$\mathrm{PL}^{\text {pro }}$ inhibitors}

The BL2 loop of SARS-CoV PL ${ }^{\text {pro }}$ is important for binding inhibitors (Baez-Santos et al., 2014a; Lee et al., 2015). This loop is variable in different $\mathrm{CoV} \mathrm{PL}^{\mathrm{pro}} \mathrm{s}$. A potent inhibitor of the SARS-CoV PL ${ }^{\text {pro }}, \mathrm{N}-[(3$-fluorophenyl) methyl]-1-[(1R)-1-naphthalen-1-ylethyl] piperidine-4-carboxamide (compound $3 \mathrm{k}, \mathrm{IC}_{50}=0.15 \pm 0.01$ $\mu \mathrm{mol} / \mathrm{L}$ ) was found to have no effect on the MERS-CoV enzyme (Baez-Santos et al., 2014b). Tyr269 and Gln270 of SARS-CoV PL ${ }^{\text {pro }}$, which are important for binding this inhibitor (Baez-Santos et al., 2014a), are replaced by Glu273 and Ala275 in the MERS-CoV protease. It seems that this structural difference in the BL2 loop has a remarkable impact on the effectiveness of the inhibitor. The structure of the MERS-CoV PL ${ }^{\text {pro }}$ (C111S)-Ub complex presented here will facilitate virtual screening of chemical libraries for specific anti - MERS-CoV PL ${ }^{\text {pro }}$ inhibitors (Hilgenfeld, 2014).

\section{The di-Ub site of $\mathrm{PL}^{\text {pro }}$}

SARS-CoV and MERS-CoV PL ${ }^{\text {pro }}$ s can digest K48- and K63-linked (poly)ubiquitin chains and remove ISG15 from proteins covalently linked to it (Ratia et al., 2014, Baez-Santos et al., 2014b). SARS-CoV PL ${ }^{\text {pro }}$ prefers binding of $\mathrm{K} 48-\mathrm{Ub}_{2}$ and ISG15 over mono-ubiquitin (Ratia et al., 2014). All evidence suggests that on the $\mathrm{PL}^{\text {pro }}$, at least two ubiquitin-binding sites (or a binding site for diubiquitin-like molecules such as ISG15) exist. Ratia et al. (2014) proposed hypothetic models for complexes of SARS-CoV PL ${ }^{\text {pro }}$ with K48-Ub ${ }_{2}$ and ISG15. These authors identified two major hydrophobic binding sites on the PL ${ }^{\text {pro }}$ for the first $\mathrm{Ub}$ (Ub1) and the second Ub (Ub2). The binding site for Ub1 comprises Met209, Pro248, and Pro249. This hydrophobic patch is conserved in MERS-CoV PL ${ }^{\text {pro }}$, although the residues are not exactly the same. Met209 of SARS-CoV PL ${ }^{\text {pro }}$ is replaced by Val210 (region III) in MERS-CoV. Pro248 is replaced by Thr249, whereas Pro249 is conserved 
(Pro250; region V) (Figure 5). The hypothetic second binding site for Ub2 (also named "ridge" region, Ratia et al., 2014) is located to the first $\alpha$ helix ( $\alpha 2)$ in the thumb domain, including residues Phe70, His74, and Leu76 of SARS-CoV PL ${ }^{\text {pro }}$. According to a structural alignment of the SARS-CoV and MERS-CoV PL ${ }^{\text {pro }}$ s, Phe70, His74, and Leu76 are changed to Lys69, Gly73, and Val75, respectively (Figure 5). In SARS-CoV PL ${ }^{\text {pro }}$, the F70S and F70A replacements lost the affinity to $\mathrm{K} 48-\mathrm{Ub}_{2}$ and ISG15 in vitro (Ratia et al., 2014); therefore, the presence of Lys69 in MERS-CoV PL ${ }^{\text {pro }}$ instead of Phe70 strongly suggests that this enzyme should bind $\mathrm{Ub} 2$ in a different way. Bailey-Elkin et al. (2014) predicted Asn 1673 and Val1674 of the fingers subdomain (corresponding to Asn192 and Val193 in our $\mathrm{PL}^{\mathrm{pro}}$ ) as the distal Ub site of K63 di-Ub, but they found that their DUB activity data do not support their prediction for the Ub2 binding site. The structure of the MERS-CoV PL ${ }^{\mathrm{pro}}-\mathrm{Ub}$ complex reported here reveals the exact Ub1 binding site on the PL ${ }^{\text {pro }}$, but the Ub2 binding site should be identified by crystallizing the $\mathrm{PL}^{\text {pro }}$ in complex with poly- or di-ubiquitin.

In summary, the crystal structure of the MERS-CoV $\mathrm{PL}^{\mathrm{pro}}-\mathrm{Ub}$ complex provides valuable information that helps understand the multiple functions of coronavirus papain-like proteases. Mutational studies additionally highlight features of the MERS-CoV PL ${ }^{\text {pro }}$. The different substrate-binding patterns should be kept in mind when designing inhibitors for $\mathrm{PL}^{\mathrm{pro}} \mathrm{s}$ of $\mathrm{CoVs}$, even though the overall structures of these enzymes are conserved. Furthermore, it would be helpful to obtain the structure of $\mathrm{PL}^{\mathrm{pro}}$ in complex with di-Ub or ISG15 in the future.

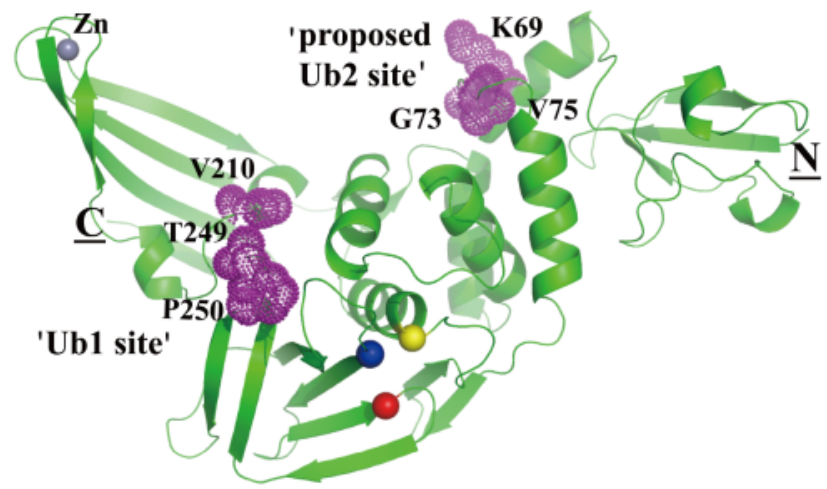

Figure 5. The two Ub-binding sites of $\mathrm{PL}^{\text {pro }}$. The Ub1 and the proposed Ub2 (according to a structural alignment with the SARS-CoV PL ${ }^{\text {pro }}$; Ratia et al., 2014) binding sites are depicted as purple dots. The $\mathrm{N}$ and $\mathrm{C}$ termini of $\mathrm{PL}^{\text {pro }}$ are marked by underlined letters. All residues related to the two $\mathrm{Ub}$ binding sites are labeled. The catalytic triad Cys111-His278-Asp293 is indicated by yellow, blue, and red spheres.

\section{ACKNOWLEDGMENTS}

Technical assistance by Susanne Zoske is gratefully acknowledged. We thank Stefan Anemüller for discussion. We also acknowledge access to beamline BL14.2 operated by the Helmholtz-Zentrum Berlin at the BESSY II electron storage ring (Berlin-Adlershof, Germany). This work was supported by the European Commission through its "SILVER" project (contract no. HEALTHF3-2010-260644) and by the German Center for Infection Research (DZIF). RH acknowledges support by the DFG Cluster of Excellence "Inflammation at Interfaces" (EXC 306)

\section{COMPLIANCE WITH ETHICS GUIDELINES}

This article does not contain any studies with human or animal subjects. Both authors declare no competing interest.

\section{AUTHOR CONTRIBUTIONS}

$\mathrm{JL}$ and RH designed all experiments. JL performed the experiments. JL and $\mathrm{RH}$ analyzed all data. JL and $\mathrm{RH}$ wrote the manuscript.

Supplementary figure and table are available on the websites of Virologica Sinica: www.virosin.org; link. springer.com/ journal/12250.

\section{REFERENCES}

Assiri A, McGeer A, Perl TM, Price CS, Al Rabeeah AA, Cummings DA, Alabdullatif ZN, Assad M, Almulhim A, Makhdoom H, Madani H, Alhakeem R, Al-Tawfiq JA, Cotton M, Watson SJ, Kellam P, Zumla AI, Memish ZA, KSA MERSCoV Investigation Team. 2013. Hospital outbreak of Middle East respiratory syndrome coronavirus. N Engl J Med, 369: 407-416.

Baez-Santos YM, Barraza SJ, Wilson MW, Agius MP, Mielech AM, Davis NM, Baker SC, Larsen SD, Mesecar AD. 2014a. Xray structural and biological evaluation of a series of potent and highly selective inhibitors of human coronavirus papain-like proteases. J Med Chem, 57: 2393-2412.

Baez-Santos YM, Mielech AM, Deng X, Baker S, Mesecar AD. 2014b. Catalytic function and substrate specificity of the PLpro domain of nsp3 from the Middle East Respiratory Syndrome Coronavirus (MERS-CoV). J Virol, 88: 12511-12527.

Bailey-Elkin BA, Knaap RC, Johnson GG, Dalebout TJ, Ninaber DK, van Kasteren PB, Bredenbeek PJ, Snijder EJ, Kikkert M, Mark BL. 2014. Crystal structure of the Middle East respiratory syndrome coronavirus (MERS-CoV) papain-like protease bound to ubiquitin facilitates targeted disruption of deubiquitinating activity to demonstrate its role in innate immune suppression. J Biol Chem, 289: 34667-34682.

Barretto N, Jukneliene D, Ratia K, Chen Z, Mesecar AD, Baker SC. 2005. The papain-like protease of severe acute respiratory 
syndrome coronavirus has deubiquitinating activity. J Virol, 79: 15189-15198.

Bekes M, Rut W, Kasperkiewicz P, Mulder MP, Ovaa H, Drag M, Lima CD, Huang TT. 2015. SARS hCoV papain-like protease is a unique Lys ${ }^{48}$ linkage-specific di-distributive deubiquitinating enzyme. Biochem J, 468: 215-226.

Butler D. 2015. South Korean MERS outbreak spotlights lack of research. Nature, 522: 139-140.

Chang CK, Hou MH, Chang CF, Hsiao CD, Huang TH. 2014. The SARS coronavirus nucleocapsid protein-forms and functions. Antiviral Res, 103: 39-50.

Chen VB, Arendall WB, Headd JJ, Keedy DA, Immormino RM, Kapral GJ, Murray LW, Richardson JS, Richardson DC. 2010. MolProbity: all-atom structure validation for macromolecular crystallography. Acta Crystallogr D Biol Crystallogr, 66: 12-21.

Chen Y, Cai H, Pan J, Xiang N, Tien P, Ahola T, Guo D. 2009. Functional screen reveals SARS coronavirus nonstructural protein nsp14 as a novel cap N7 methyltransferase. Proc Natl Acad Sci USA, 106: 3484-3489.

Chou CY, Lai HY, Chen HY, Cheng SC, Cheng KW, Chou YW. 2014. Structural basis for catalysis and ubiquitin recognition by the severe acute respiratory syndrome coronavirus papain-like protease. Acta Crystallogr D Biol Crystallogr, 70: 572-581.

Dikic I, Wakatsuki S, Walters KJ. 2009. Ubiquitin-binding domains-from structures to functions. Nat Rev Mol Cell Biol, 10: 659-671.

Drosten C, Günther S, Preiser W, van der Werf S, Brodt HR, Becker S, Rabenau H, Panning M, Kolesnikova L, Fouchier RA, Berger A, Burguiere AM, Cinatl J, Eickmann M, Escriou N, Grywna K, Kramme S, Manuguerra JC, Müller S, Rickerts V, Stürmer M, Vieth S, Klenk HD, Osterhaus AD, Schmitz H, Doerr HW. 2003. Identification of a novel coronavirus in patients with severe acute respiratory syndrome. N Engl J Med, 348: 1967-1976.

Eckerle I, Müller MA, Kallies S, Gotthardt DN, Drosten C. 2013. In-vitro renal epithelial cell infection reveals a viral kidney tropism as a potential mechanism for acute renal failure during Middle East Respiratory Syndrome (MERS) coronavirus infection. Virol J, 10: 359.

Emsley P, Lohkamp B, Scott WG, Cowtan K. 2010. Features and development of Coot. Acta Crystallogr D Biol Crystallogr, 66: 486-501.

Fetics SK, Guterres H, Kearney BM, Buhrman G, Ma B, Nussinov R, Mattos C. 2015. Allosteric effects of the oncogenic RasQ61L mutant on Raf-RBD. Structure, 23: 505-516.

Frieman M, Ratia K, Johnston RE, Mesecar AD, Baric RS. 2009. Severe acute respiratory syndrome coronavirus papain-like protease ubiquitin-like domain and catalytic domain regulate antagonism of IRF3 and NF- $\kappa$ B signaling. J Virol, 83: 6689-6705.

Hale BG, Randall RE, Ortin J, Jackson D. 2008. The multifunctional NS1 protein of influenza A viruses. J Gen Virol, 89: 2359-2376.

Hamre D, Procknow JJ. 1966. A new virus isolated from the human respiratory tract. Proc Soc Exp Biol Med, 121: 190-193.

Harcourt BH, Jukneliene D, Kanjanahaluethai A, Bechill J, Severson KM, Smith CM, Rota PA, Baker SC. 2004. Identification of severe acute respiratory syndrome coronavirus replicase products and characterization of papain-like protease activity. $\mathrm{J}$ Virol, 78: 13600-13612.

Headd JJ, Echols N, Afonine PV, Grosse-Kunstleve RW, Chen VB, Moriarty NW, Richardson DC, Richardson JS, Adams PD. 2012. Use of knowledge-based restraints in phenix.refine to improve macromolecular refinement at low resolution. Acta Crystallogr D Biol Crystallogr, 68: 381-390.
Hilgenfeld R. 2014. From SARS to MERS: Crystallographic studies on coronaviral proteases enable antiviral drug design. FEBS J, 281: 4085-4096.

Hilgenfeld R, Peiris M. 2013. From SARS to MERS: 10 years of research on highly pathogenic human coronaviruses. Antiviral Res, 100: 286-295.

Hu M, Li P, Song L, Jeffrey PD, Chenova TA, Wilkinson KD, Cohen RE, Shi Y. 2005. Structure and mechanisms of the proteasome-associated deubiquitinating enzyme USP14. EMBO J, 24: 3747-3756.

Kabsch W. 2010.XDS. Acta Crystallogr D Biol Crystallogr, 66: $125-132$

Krissinel E, Henrick K. 2007. Inference of macromolecular assemblies from crystalline state. J Mol Biol, 372: 774-797.

Ksiazek TG, Erdman D, Goldsmith CS, Zaki SR, Peret T, Emery S, Tong S, Urbani C, Comer JA, Lim W, Rollin PE, Dowell SF, Ling AE, Humphrey CD, Shieh WJ, Guarner J, Paddock CD, Rota P, Fields B, DeRisi J, Yang JY, Cox N, Hughes JM, LeDuc JW, Bellini WJ, Anderson LJ, SARS Working Group. 2003. A novel coronavirus associated with severe acute respiratory syndrome. N Engl J Med, 348: 1953-1966.

Kuiken T, Fouchier RA, Schutten M, Rimmelzwaan GF, van Amerongen G, van Riel D, Laman JD, de Jong T, van Doornum G, Lim W, Ling AE, Chan PK, Tam JS, Zambon MC, Gopal R, Drosten C, van der Werf S, Escriou N, Manuguerra JC, Stöhr K, Peiris JS, Osterhaus AD. 2003. Newly discovered coronavirus as the primary cause of severe acute respiratory syndrome. Lancet, 362: 263-270.

Lee H, Lei H, Santarsiero BD, Gatuz JL, Cao S, Rice AJ, Patel K, Szypulinski MZ, Ojeda I, Ghosh AK, Johnson ME. 2015. Inhibitor recognition specificity of MERS-CoV papain-like protease may differ from that of SARS-CoV. ACS Chem Biol, 10: 1456-1465.

Lei J, Mesters JR, Drosten C, Anemüller S, Ma Q, Hilgenfeld R. 2014. Crystal structure of the papain-like protease of MERScoronavirus reveals unusual, potentially druggable active-site features. Antiviral Res, 109: 72-82.

Liu YC, Penninger J, Karin M. 2005. Immunity by ubiquitylation: a reversible process of modification. Nat Rev Immunol, 5: 941-952.

Maringer K, Fernandez-Sesma A. 2014. Message in a bottle: lessons learned from antagonism of STING signalling during RNA virus infection. Cytokine Growth Factor Rev, 25: 669-679.

McIntosh K, Becker WB, Chanock RM. 1967. Growth in suckling-mouse brain of "IBV-like" viruses from patients with upper respiratory tract disease. Proc Natl Acad Sci USA, 58: 2268-2273.

Mielech AM, Kilianski A, Baez-Santos YM, Mesecar AD, Baker SC. 2014. MERS-CoV papain-like protease has delSGylating and deubiquitinating activities. Virology, 450-451: 64-70.

Mielech AM, Deng X, Chen Y, Kindler E, Wheeler DL, Mesecar AD, Thiel V, Perlman S, Baker SC. 2015. Murine coronavirus ubiquitin-like domain is important for papain-like protease stability and viral pathogenesis. J Virol, 89: 4907-4917.

Mueller U, Darowski N, Fuchs MR, Förster R, Hellmig M, Paithankar KS, Pühringer S, Steffien M, Zocher G, Weiss MS. 2012. Facilities for macromolecular crystallography at the Helmholtz-Zentrum Berlin. J Synchrotron Radiat, 19: 442-449.

Peiris JS, Lai ST, Poon LL, Guan Y, Yam LY, Lim W, Nicholls J, Yee WK, Yan WW, Cheung MT, Cheng VC, Chan KH, Tsang DN, Yung RW, Ng TK, Yuen KY, SARS Study Group. 2003. Coronavirus as a possible cause of severe acute respiratory syndrome. Lancet, 361: 1319-1325.

Pfoh R, Lacdao IK, Georges AA, Capar A, Zheng H, Frappier L, 
Saridakis V. 2015. Crystal structure of USP7 ubiquitin-like domains with an ICP0 peptide reveals a novel mechanism used by viral and cellular proteins to target USP7. PLoS Pathog, 11: e1004950.

Ratia K, Kilianski A, Baez-Santos YM, Baker SC, Mesecar AD. 2014. Structural basis for the ubiquitin-linkage specificity and deISGylating activity of SARS-CoV papain-like protease. PLoS Pathog, 10: e1004113.

Vagin A, Teplyakov A. 2010. Molecular replacement with MOLREP. Acta Crystallogr D Biol Crystallogr, 66: 22-25.

van der Hoek L, Pyrc K, Jebbink MF, Vermeulen-Oost W, Berkhout RJ, Wolthers KC, Wertheim-van Dillen PM, Kaandorp J, Spaargaren J, Berkhout B. 2004. Identification of a new human coronavirus. Nat Med, 10: 368-373.

Vijay-Kumar S, Bugg CE, Cook WJ. 1987. Structure of ubiquitin refined at $1.8 \AA$ resolution. J Mol Biol, 194: 531-534.

Wang H, Xue S, Yang H, Chen C. 2016. Recent progress in the discovery of inhibitors targeting coronavirus proteases. Virol Sin, 31: 24-30.

Weiss MS, Hilgenfeld R. 1997. On the use of the merging $R$ factor as a quality indicator for X-ray data. J Appl Cryst, 30: 203-205. Wojdyla JA, Manolaridis I, van Kasteren PB, Kikkert M, Snijder EJ, Gorbalenya AE, Tucker PA. 2010.Papain-like protease 1 from transmissible gastroenteritis virus: crystal structure and enzymatic activity toward viral and cellular substrates. J Virol, 84: 10063-10073.

Woo PC, Lau SK, Chu CM, Chan KH, Tsoi HW, Huang Y, Wong BH, Poon RW, Cai JJ, Luk WK, Poon LL, Wong SS, Guan Y, Peiris JS, Yuen KY. 2005. Characterization and complete genome sequence of a novel coronavirus, coronavirus HKU1, from patients with pneumonia. J Virol, 79: 884-895.

Yang X, Chen X, Bian G, Tu J, Xing Y, Wang Y, Chen Z. 2013. Proteolytic processing, deubiquitinase and interferon antagonist activities of Middle East respiratory syndrome coronavirus papain-like protease. J Gen Virol, 95: 614-626.

Zaki AM, van Boheemen S, Bestebroer TM, Osterhaus AD, Fouchier RA. 2012. Isolation of a novel coronavirus from a man with pneumonia in Saudi Arabia. N Engl J Med, 367: 18141820. 